\title{
Peculiarities of Arterial and Venous Hemodynamics with Transitorial Ischemic Attacks in the Vertebro-Basilary Basin
}

\author{
Abdullaiev R.Ya*., Kalashnikov VI, Sysohn LA, Abdullaiev R.R. \\ *Head of the Department of Ultrasound Diagnostics of Kharkov Medical Academy of Postgraduate \\ Education,Ukraine \\ r.abdullaev@bk.ru \\ rizvanabdullaiev@gmail.com
}

Objective: Ultrasonic dopplerographic examination of arterial and venous hemodynamics in patients with transient ischemic attacks (TIA) in the vertebrobasilar basin.

Materials and Methods: 86 patients of a young age (18-35 years) with a clinical picture of transient ischemic attacks (TIA) were examined. Clinically, TIAs were manifested in the form of cochlear-vestibular (CV) and syncopal vertebral (SV) syndromes. All patients complained of cervicogenic paine. In the studied persons dominated the occipital localization of pain (87.6\%). The pulsating type of pain was observed in $21.4 \%$ of patients, bursting - in $52,8 \%$, their combination - in $25.7 \%$.

The investigation was carried out in triplex dopplerographic mode. The parameters of hemodynamics in the basilar and vertebral arteries (BA and VA), vertebral and basilar veins (VV and BV), direct sinus (DS) were studied.

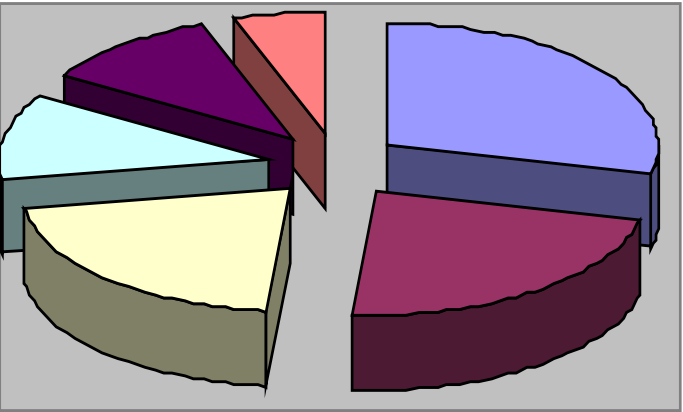

$\square$ VA stenosis
$\square$ VA compression
$\square$ VA/BA
Vasospasm
$\square$ VA stroke
abnormalities
$\square$ VA hypoplasia
$\square$ Styl-syndrome

Figure1. The frequency of detection of TIA vascular pathogenetic factors (\%). 
Peculiarities of Arterial and Venous Hemodynamics with Transitorial Ischemic Attacks in the Vertebro-Basilary Basin

\section{RESULTS AND DISCUSSION}

The frequency of detection of TIA vascular pathogenetic factors was as follows: VA stenosis in 37,6\% cases, vertebral artery compression in $29.8 \%$, vasospasm in VA and / or BA in $27.6 \%$ cases, VA stroke abnormalities in $14.8 \%$ cases. Hypoplasia of the VA - $13.9 \%$, styl-syndrome - in $7.3 \%$ of cases. Some of the patients had a combination of these factors. The occurrence of $\mathrm{CV}$ was almost equally caused by stenosis, vertebral compression and VA vasospasm $(36.7 \%, 34.4 \%$ and $31.5 \%$ of patients, respectively). In patients with SVS, combinations of vertebral compressions with stroke anomalies (41.6\%) or hypoplasia (36.1\%) of vertebral arteries were most often detected. Disturbances of venous hemodynamics in a significant majority of patients with TIA (72.3\%) were observed in the VV in the form of an increase in systolic blood flow velocity to $35 \mathrm{~cm} / \mathrm{s}$. In the group of patients with cochlear-vestibular syndrome also increased blood flow in the DS (44.2\% of patients) and BV (29.4\%). In $68.3 \%$ of patients with syncopal vertebral syndromes, a combination of an increase in outflow in the pylori with an asymmetry of systolic blood flow velocity for VV (25-30\%) was revealed. Infringements of a blood flow in DS and BV were observed accordingly at $14,6 \%$ and $10,1 \%$ of patients.

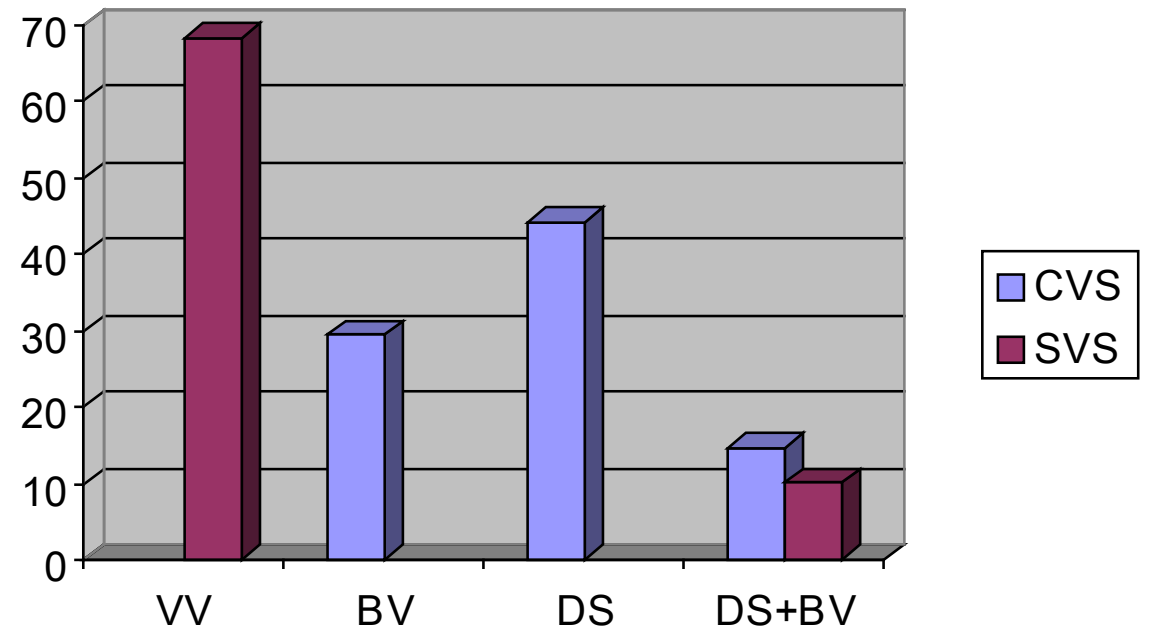

Figure2. The frequency of venous hemodynamics disturbances in patients with TIA (\%).

\section{CONCLUSION}

The leading hemodynamic factors in the development of transient ischemic attacks in vertebrobasillar basin are stenosis, vertebral compression and vertebral artery vasospasm, which are manifested equally in cochlearvestibular syndromes (CVS). Vertebral compression is more conducive to the development of syncopal vertebral syndromes (SVS). Disturbances of venous hemodynamics in CVS were manifested in the form of dyscirculation in VV and direct sinus, and in SVS mainly as a combination of outflow enhancement and asymmetry in vertebral vein.

\section{REFERENCE}

1. Baird TA, Muir KW, Bone I. Basilar artery occlusion. Neurocritical care. 2004;3:319-30. pubmed

2. Savitz SI, Caplan LR. Vetebrobasilar disease. N Engl J Med. 2005;352:2618-26. pubmed 
Peculiarities of Arterial and Venous Hemodynamics with Transitorial Ischemic Attacks in the Vertebro-Basilary Basin

3. Cassidy J.D., Boyle E., Cote P., at al. Risk of vertebrobasilar stroke and chiroptactic care: results of population-based case-control and case-crossover study. J Manipulative physiol Ther. 2009. Feb; 32 (2 Suppl): S 201-8.doi:10.1016/j.jmt.2008.11.020

4. Ghezzi A, Comi G, Federico A. Chronic cerebrospinal venous insufficiency (CCSVI) and multiple sclerosis. Neurol Sci 2010;32(1):17-21.

5. Khan O, Filippi M, Freedman MS, et al. Chronic cerebrospinal venous insufficiency and multiple sclerosis. Ann Neurol 2010;67(3):286-90.

6. Simka M, Kostecki J, Zaniewski M, Majewski E, Hartel M. Extracranial Doppler sonographic criteria of chronic cerebrospinal venous insufficiency in the patients with multiple sclerosis. Int Angiol 2010;29(2):109-14.

7. Hojnacki D, Zamboni P, Lopez -Soriano A, et al. Use of neck magnetic resonance venography, Doppler sonography and selective venography for diagnosis of chronic cerebrospinal venous insufficiency: a pilot study in multiple sclerosis patients and healthy controls. Int Angiol 2010;29(2):127-39.

8. Bastianello S, Romania A, Viselner G, et al. Chronic cerebrospinal venous insufficiency in multiple sclerosis: clinical correlates from a multicentre study. BMC Neurology 2011;11:132.

9. Iris Zavoreo, Vanja Baљić-Kes, Lucija Zadro-Matovina, et al. Cerebral venous circulatory system by ultrasonography. Acta Clin Croat 2013; 52:203-211.

10. Abdullaev R.Ya., Sysun L.A., Marchenko V.G., Kalashnikov V. Changes in venous blood flow in the first day of a semi-latered ischemic stroke according to transcranial dopplerography data. Materials of the educational-scientific conference "Modern postgraduate education: achievements, problems, perspectives" devoted to the 90th anniversary of the establishment of KhMAPO. November 7-8, 2013, Kharkiv. - P. 211.

11. Abdullaev R.Ya., Kalashnikov VI, Sysohn L.A. Dopplerographic patterns in transient ischemic attacks in the vertebrobasilar basin. Promeneva dignostyka, promeneva terapiya, 2014, №1-2, P. 122.

12. Abdullaeiev R. Y., Khvisyuk A. N., Marchenko V. G. Echography in Neurology and Vertebrology (book). Ukraine, Kharkov, "Fact”, 2017, 196p.

13. Abdullaeiev R. Y., Marchenko V. G. Fedko L.A. Sysun. Dopplerography with ischemic stroke. A Handbook, H.Nove Word, 2012, 116 p.

Citation: Abdullaiev R.Ya, Kalashnikov VI, Sysohn LA, Abdullaiev R.R. "Peculiarities of Arterial and Venous Hemodynamics with Transitorial Ischemic Attacks in the Vertebro-Basilary Basin ". American Research Journal of Neurology; V1, I1; pp: 4-6

Copyright (c) 2017 Abdullaiev R.Ya., Kalashnikov VI, Sysohn LA, Abdullaiev R.R, This is an open access article distributed under the Creative Commons Attribution License, which permits unrestricted use, distribution, and reproduction in any medium, provided the original work is properly cited. 